\title{
Análisis Cualitativo de la empresa
}

Javier JARA RENGIFO

\section{RESUMEN}

El análisis de empresas se realiza para evaluar la situación actual y perspectivas de la empresa con el fin de tomar decisiones adecuadas conducente a alcanzar mejor sus objetivos.

El análisis integral de empresas comprende: el análisis cualitativo, es decir, el análisis de la información no financiera; y el análisis de la información financiera, esto es, el análisis e interpretación de estados financieros.

Para realizar el análisis integral de una empresa se puede tener en cuenta las siguientes cinco partes:

1. Situación y perspectivas del entorno político, económico y social.

2. Debilidades, amenazas, fortalezas, oportunidades - DAFO.

3. Diagrama causa - efecto.

4. Recomendaciones.

5. Demostración de la conveniencia de las recomendaciones.

El análisis cualitativo de la empresa discurre en los factores relacionados con QUIÉN, esto es, con las personas que integran la empresa, QUÉ hace la empresa (problemas estratégicos) y, CÓMO lo hace la empresa (problemas operativos). Con el análisis cualitativo se consigue una primera visión de la situación y perspectivas de la empresa que se deberá completar con el análisis e interpretación de los estados financieros (análisis de los aspectos cuantitativos).

\section{PALABRAS CLAVES}

Análisis cualitativo. Análisis cuantitativo. Problemas de personas. Problemas estratégicos. Problemas operativos. Estados Financieros.

\section{INTRODUCCIÓN AL ANÁLISIS INTEGRAL DE EMPRESAS} El análisis integral de empresas comprende el ANÁLISIS CUALITATIVO, es decir, el análisis de la información no financiera; y el ANÁLISIS DE LA 
INFORMACIÓN FINANCIERA, esto es, el análisis e interpretación de estados financieros.

El análisis de una empresa se realiza para evaluar su historia (pasado), su situación actual (presente) y las perspectivas de futuro con el fin de poder tomar decisiones adecuadas con relación a la empresa.

El análisis integral de la empresa no solamente interesa a sus directivos, sinó también a cualquier persona que tenga relaciones con la empresa tales como: bancos, accionistas, proveedores, trabajadores, competidores, entre otros.

Conocer mejor la empresa ayuda a alcanzar los objetivos, ya sea ganar más dinero, proporcionar productos y servicios de mejor calidad o contribuir a un mundo mejor. Con un diagnóstico acertado de la situación en que se encuentra la empresa se puede poner en marcha aquellas situaciones que corrijan los puntos débiles que pueden amenazar su futuro, al mismo tiempo que se saca provecho de los puntos fuertes para que la empresa alcance sus objetivos.

\section{PARTES DEL ANÁLISIS DE EMPRESAS}

Para realizar el análisis integral de una empresa se puede tener en cuenta las siguientes cinco partes:

a) Situación y perspectivas del entorno político, económico y social. Antes de proceder al análisis de una empresa conviene tener clara la situación y perspectivas económicas, sociales, y políticas, ya que influyen decisivamente en la evolución actual y futura de cualquier organización. Para ello, hay que analizar los siguientes temas, entre otros.

\section{- Estabilidad política}

Cuando la situación política y social es estable y sin sobresaltos, se dan mejores condiciones para que la economía y las empresas funcionen mejor.

\section{- Actitud gubernamental hacia la empresa}

Cuando los gobiernos tienen dirigentes interesados en la buena marcha de la economía y de las empresas suelen aprobarse leyes fiscales y económicas que impulsan el crecimiento económico.

\section{- Producto Bruto Interno (PBI)}

Para tener una aproximación de la evolución del nivel de actividad y, por tanto, de la posibilidad de recesión o expansión de la economía.

\section{- Índice de Precios del Consumidor (IPC)}

Puede influir en el nivel de beneficio, sobre todo cuando los incrementos de gastos no pueden ser repercutidos vía precio. 


\section{- Tasa de desempleo}

Ayuda a visualizar la tendencia de la economía y puede anticipar la facilidad o dificultad de encontrar empleados.

- Tipos de interés

El coste de la financiación si es reducido puede alentar el crecimiento de la economía. En cambio, cuando los tipos de interés son elevados se relentiza la economía.

- Tipos de cambio de las divisas

Su evolución puede influir en las exportaciones e importaciones.

- Nivel de Actividad

En sectores que son motores de la economía, vivienda, automóvil, turismo.

\section{- Riesgo de crédito}

Morosidad de las entidades de crédito, evolución de impagados.

Cuando se tenga una idea clara de si se está en recesión o en un momento de bonanza económica y si se cree que la situación general va a mejorar o a empeorar en el futuro inmediato, ya se puede iniciar el análisis de la empresa.

\section{b) Debilidades, amenazas, fortalezas, oportunidades. DAFO}

$\mathrm{Al}$ analizar una empresa se trata de obtener un diagnóstico de sus fortalezas, y debilidades, las fortalezas son aquellas bases que se pueden utilizar para afrontar con mejores posibilidades el futuro, tales como: tener sobrantes de tesorería, productos muy solicitados por los clientes, una buena calidad de servicio, una imagen apreciada por los clientes, la elevada motivación de los trabajadores, o en unas buenas infraestructuras de producción. Las debilidades son las que se deben corregir para evitar que la empresa tenga problemas graves que puedan limitar su evolución o incluso que pueden provocar su cierre. Ejemplo: falta de dinero, gastos excesivos, ventas insuficientes.

Además, las fortalezas y debilidades se complementan con un análisis del sector y el mercado para identificar las amenazas, y las oportunidades. Las amenazas, tales como: entrada de nuevos competidores, la globalización de los mercados, dificultad en encontrar mano de obra cualificada, nuevas normativas que pueden perjudicar a la empresa, constituyen peligros que se avecinan y que conviene afrontar sacando partido de las oportunidades que la empresa puede aprovechar. Ejemplo: incremento de la demanda por cambios en el mercado, un potencial lanzamiento de nuevos productos y servicio aprovechando sinergias que tiene la empresa, entre otros. 


\section{c) Diagrama causa - efecto}

La relación de debilidades - amenazas - fortalezas - oportunidades, se puede esquematizar con el denominado diagrama causa - efecto, que empieza identificando los principales puntos fuertes y débiles de la empresa que tienen consecuencia en otras partes de la empresa. Por ejemplo, si una empresa por una mala política de innovación (causa) tiene productos poco competitivos y consecuente ventas insuficientes y, además, tiene gastos excesivos, es posible que tenga pérdida (efecto). Si encima se financia con un exceso de deudas a corto plazo (causa) puede tener dificultades para atender sus pagos y verse abocada a una suspensión de pago (efecto).

El diagrama causa - efecto de este ejemplo va de arriba abajo que a continuación se describe.

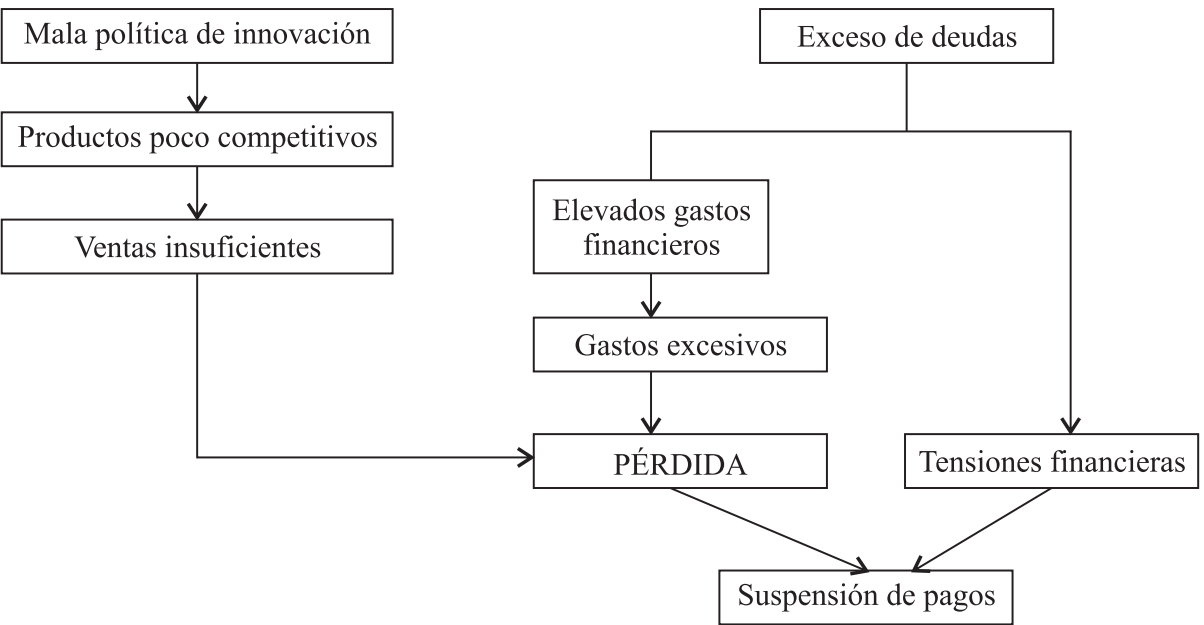

\section{d) Recomendaciones}

Realizado el diagrama causa - efecto, ya se puede formular más fácilmente las recomendaciones que pueden solucionar las principales debilidades de la empresa. Para ello, se trata de combatir a tiempo las amenazas y, aprovechar las oportunidades que se presentan. Por ejemplo, de acuerdo al caso precedente de causa y efecto se podrían formular las siguientes recomendaciones:

\section{Mala política de innovación}

Invertir 300,000 dólares en innovación con el fin de conseguir productos competitivos. 


\section{Ventas insuficientes}

Elaborar un nuevo plan de marketing con una inversión de 200,000 dólares en promoción y publicidad para aumentar las ventas en un $10 \%$.

\section{Exceso de deudas}

Ampliar el capital en 100,000 dólares y vender por 400,000 dólares un edificio que no se necesita para la actividad para reducir las deudas y así reducir los gastos financieros.

\section{Gastos excesivos}

Analizar todos los gastos para ver los que son susceptibles de reducción sin que se resienta la calidad de la empresa que podría ser recortar un $15 \%$ los gastos de estructura.

Si estas recomendaciones son eficaces se incrementarán los beneficios y se podrán atender las deudas con lo que se alejará el peligro de suspender pagos.

\section{e) Demostración de la conveniencia de las recomendaciones}

Laúltima parte del análisis consiste en demostrar que con las recomendaciones propuestas la empresa mejorará su situación y estará en mejores condiciones de alcanzar sus objetivos. Esto implica cuantificar los efectos previsibles de las recomendaciones en la rentabilidad y la solvencia de la empresa.

\section{ANÁLISIS CUALITATIVO DE LA EMPRESA}

¿Cuáles son las diferencias entre las empresas exitosas y las que fracasan? El análisis de muchas empresas, entre las que se incluyen empresas exitosas y empresas que han fracasado, permite detectar factores cualitativos (estratégicos y operativos) que aumentan la probabilidad de éxito de un proyecto empresarial. Estos factores cualitativos se pueden dividir entre los que están relacionados con QUIÉN, esto es, con las personas que integran la empresa y, los que tienen que ver con lo QUÉ hace la empresa y, finalmente, los que nos informan de CÓMO lo hace.

\subsection{QUIÉN: El empresario, el equipo directivo y las personas que forman parte de la empresa.}

Existen factores de éxito que están directamente relacionados con la representación que encarna el empresario, el directorio, la gerencia y los trabajadores de la empresa, estos factores son los siguientes:

a) Grado de implicación en el proyecto. Los emprendedores y directivos de empresas exitosas se caracterizan por una apuesta total, es decir, su empresa forma parte de un proyecto de vida. Este grado de implicación se manifiesta con una dedicación que muchas veces supera las cincuenta horas de trabajo a la semana. 
b) Tienen un nivel de formación adecuado. Por tanto, los procesos de selección y los planes de formación son importante, lo cual se perciben como una inversión fundamental.

c) Cuentan con conocimiento suficiente del sector. Por lo tanto, detectan las fuentes de ventajas competitivas que permitirán que la empresa sea mejor que sus competidores.

d) Habilidades directivas. Saben escuchar, dirigir reuniones y son buenos negociadores.

e) Son personas que se fijan objetivos a largo plazo y que toman decisiones pensando en el futuro y no en los beneficios inmediatos.

f) Tienen sistemas de incentivos adecuados para la motivación de todas las personas que trabajan en la empresa, lo cual conduce a un buen clima laboral.

Para poder evaluar estos aspectos referidos a las personas que tiene la empresa es imprescindible conocerlas y también conviene pedir referencias a otras personas que las conozcan, tales como: bancos, proveedores, clientes, entre otros.

\section{2 ¿QUÉ hace la empresa?: aspectos estratégicos.}

Un segundo grupo de consideraciones a tener en cuenta al evaluar los elementos cualitativos está relacionado con QUÉ hace la empresa:

a) Sector o sectores de actividad en los que actúa la empresa.

b) Barreras de entrada, tales como: las patentes, la tecnología, el capital necesario, la red de distribución.

c) Mercados geográficos en los que actúa la empresa: Cuanto mayor sea la diversificación geográfica, más se diluye el riesgo.

d) Productos y servicios que ofrece la empresa: Se trata de evaluar si la empresa es exitosa innovando y si los productos tienen demanda suficiente en la actualidad y en el futuro.

e) Plan estratégico: Objetivos a alcanzar a mediano y largo plazo, y planes para conseguirlo.

f) Dependencias: Una sujeción excesiva de algo o de alguien puede poner en peligro la vida de la empresa cuando se produce algún problema. Por ejemplo, si un cliente representa más del 20\% de la facturación, y por cualquier motivo se pierde dicho cliente, será difícil que la empresa sobreviva.

g) Poder de negociación con clientes, proveedores, bancos, trabajadores, entre otros: El poder de negociación es una de las claves para evolucionar favorablemente en el futuro. 
h) Ventajas competitivas: Las que se traduce en costes, en imagen y prestigio, en proximidad al cliente.

\section{3 ¿CÓMO lo hace la empresa?: aspectos operativos.}

Está referido básicamente a temas, tales como: procesos, clientes y finanzas que a continuación se desarrolla:

a) Procesos: La excelencia en los procesos (de innovación, compras, producción, calidad total, logística, comercialización, servicio post-venta, administración, ...) requiere diversos ingredientes, como: la búsqueda de la calidad total, la innovación y la flexibilidad.

b) Clientes: Las características anteriores deberán revertir en una mayor satisfacción y fidelidad de los clientes y, en definitiva, en mayores ventas.

c) Finanzas: Si la empresa es exitosa en temas como los indicados, obtendrá resultados financieros adecuados. Para ello debería gestionar de forma eficiente los activos (gracias a la mejor utilización de los mismos), y un incremento de los beneficios (gracias al incremento de las ventas y a la reducción de los gastos), lo cual debe plasmarse en incrementos de la riqueza generada.

\section{INFORMACIÓN MÍNIMA NECESARIA PARA EL ANÁLISIS CUALITATIVO}

\section{a) Sector}

Situación y perspectivas del sector

Situación y movimientos de los principales competidores

b) Antecedentes y datos legales

Historia

Sede social y sucursales

Escritura de constitución, estatutos.

Relación de accionistas

Componentes de los órganos de dirección de la empresa.

Participaciones en otras empresas.

c) Aspectos generales

Plan estratégico.

Perfil de los fundadores de la empresa.

Cultura de la empresa.

Organigrama y principales directivos.

Compromisos contractuales con otras empresas.

d) Innovación, producción, logística y calidad

Investigación y desarrollo.

Capacidad para lanzar nuevos productos al mercado. 
Proceso de producción.

Productividad.

Niveles de ocupación de la capacidad productiva.

Estado de los equipos.

Peritaje por experto independiente de los activos inmovilizados.

Materias primas, componentes y proveedores.

Subcontratación.

Mantenimiento.

Calidad total: actividades de prevención y evaluación.

No calidad: errores, reclamaciones, ...

Actuaciones en materia de prevención y evaluación de la calidad., Errores y costes de la mala calidad.

Inversiones y actuaciones en materia de medio ambiente.

Costes logísticos.

Plazos de entrega.

e) Marketing y ventas

Plan de marketing.

Precios, descuentos, formas de cobro.

Catálogo de productos y servicios.

Evolución de venta por familia, productos, mercados geográficos.

Cuota de mercado.

Listado de clientes.

Publicidad y promoción.

f) Administración y Finanzas.

Dictamen de los auditores independientes y correspondientes estados financieros.

Entidades de crédito.

Opiniones que tienen los bancos sobre la empresa.

Clientes y proveedores con que opera y opiniones que tienen estos sobre la empresa. Condiciones de pago a proveedores y de cobro a clientes.

Informes comerciales.

Deudas por impuestos.

Informes de la Central de Riesgos.

Política de prevención de riesgo (comerciales, incendios, robo, tipo de cambio, tipos de intereses, ...).

Presupuestos a corto y largo plazo de la empresa.

g) Sistemas de Información.

Equipamiento informático.

Programas informáticos

Sistema de información integrada

Cuadros de mando 
h) Recursos Humanos

Política de selección y de formación

Sistemas de incentivos

Clima laboral

Salud laboral

Promoción

i) Aspectos jurídicos

Percances y litigios

Sentencias pendientes de ejecución, sentencias por notificar, ...

\section{CAUSAS DE LOS FRACASOS EMPRESARIALES}

Las empresas que suspenden pagos o quiebran suelen tener importantes deficiencias internas como las que siguen:

\subsection{Problemas de las personas (QUIÉN):}

Conflictos entre accionistas, o entre directivos.

Conflictividad laboral.

Falta de liderazgo.

Mala selección de empleados.

Formación insuficiente o errónea.

Ausencia de política de incentivos o política errónea.

Empleados desmotivados.

Desconocimiento de cómo funciona el sector.

Carencias de habilidades directivas.

Arrogancia. Sensación de que ya se ha alcanzado el éxito, ya que como se dice a menudo "cuando crees que eres alguien ya no eres nadie".

Pesimismo.

Cortoplacismo que reparte demasiados dividendos y demasiado pronto; y que dificulta buscar relaciones duraderas con clientes, empleados, proveedores,...

\subsection{Problemas estratégicos (QUÉ HACE LA EMPRESA):}

Sector con malas perspectivas y con muchos competidores.

Plan estratégico poco concreto, o irrealizable o mal planteado.

Desconocimiento entre muchos de los colaboradores del plan estratégico.

Productos obsoletos.

Exceso de diversificación.

Fracasos masivos en el lanzamiento de nuevos productos.

\subsection{Problemas operativos (CÓMO LO HACE LA EMPRESA):}

Procesos

Insuficiente innovación. 
Mala selección de proveedores o precios de compra demasiados altos.

Falta de flexibilidad.

Problemas de calidad: errores, devoluciones, quejas de clientes.

Errores de logística.

Diseño erróneo de la política de marketing (precios de venta demasiado altos o demasiado bajos, publicidad insuficiente o mal planteada, ausencia de premoción).

Mal servicio post - venta.

\section{Clientes}

Clientes insatisfechos

Falta de fidelidad de la clientela.

\section{Administración - finanzas}

Descontrol.

Falta de información en temas clave como contabilidad, costes, clientes, ingresos.

Política de gestión de riesgos inexistente o inadecuada en temas, tales como: protección de activos, riesgos financieros, riesgos de tipo de cambio, riesgo de morosidad.

Diseño organizativo defectuoso (organigrama, manual de funciones).

Pérdidas por exceso de costes o ingresos insuficientes.

Inversión excesiva en activos no corrientes (planta, maquinaria, equipo) y en activos corrientes (inventarios, cuentas por cobrar comerciales).

Insuficiente aportes de capital por parte de los socios o accionistas.

Crecimiento demasiado acelerado para las posibilidades financieras de la empresa.

Reparto excesivo de dividendos a los accionistas.

Los problemas citados son solamente una muestra de aquellos que pueden hacer que una empresa sea inviable. Cualquiera de las deficiencias mencionadas pueden provocar el fin de la empresa si no se soluciona a tiempo.

\section{BIBLIOGRAFÍA}

Oriol Amat. Análisis Integral de Empresas - Claves para un chequeo completo: desde el análisis cualitativo al análisis de balances. Edición 2012. Alfaomega. México. 